\title{
J oint TAS and Power Allocation for IHDAF Relaying M2M Cooperative Networks
}

\author{
Lingwei Xu ${ }^{1}$, Hao Zhang ${ }^{2,3}$, and T. Aaron Gulliver ${ }^{3}$ \\ ${ }^{1}$ Department of Information Science and Technology, Qingdao University of Science \& Technology \\ Qingdao 266061-China \\ [e-mail: gaomilaojia2009@163.com] \\ ${ }^{2}$ College of Information Science and Engineering, Ocean University of China \\ Qingdao 266100-China \\ [e-mail: zhanghao@ouc.edu.cn] \\ ${ }^{3}$ Department of Electrical and Computer Engineering, University of Victoria \\ Victoria V8W 2 Y2 - Canada \\ [e-mail: agullive@ece.uvic.ca] \\ *Corresponding author: Lingwei Xu
}

Received October 22, 2015; revised December 29, 2015; accepted April 3, 2016; published May 31, 2016

\begin{abstract}
The outage probability (OP) performance of multiple-relay-based incremental hybrid decode-amplify-forward (IHDAF) relaying mobile-to-mobile (M2M) networks with transmit antenna selection (TAS) over $N$-Nakagami fading channels is investigated in this paper. The closed-form expressions for approximate OP of the optimal and suboptimal TAS schemes are derived. The power allocation problem is formulated for performance optimization. Then the OP performance under different conditions is evaluated through numerical simulations to verify the analysis. The simulation results showed that optimal TAS scheme has a better OP performance than suboptimal TAS scheme; the power-allocation parameter has an important influence on the OP performance.
\end{abstract}

Keywords: M2M communication, $N$-Nakagami fading channels, incremental hybrid decode-amplify-forward, outage probability, transmit antenna selection, power allocation

This research was supported by National Natural Science Foundation of China (No. 61304222, No. 60902005), Natural Science Foundation of Shandong Province (No.ZR2012FQ021), Shandong Province Higher Educational Science and Technology Program (No.J12LN88), Shandong Province Outstanding Young Scientist Award Fund (Grant No. 2014BSE28032). 


\section{Introduction}

In recent years, with the increasing demand for high-data-rate services,mobile-to-mobile (M2M) communication has attracted wide research interest [1]. In M2M communication scenarios, mobile user equipments can directly communicate to each other without the relay of base station. In particular, compared to the traditional cellular communication mode, it requires only half of the resources, thus it can offer better spectral efficiency and reduce the traffic load of the core network [2]. M2M communication has many advantages, such as increasing data rate, reducing energy cost, reducing transmission delays, and extending coverage area. M2M communication can enable new peer-to-peer and location-based applications and services. Due to its advantages, M2M communication is widely employed in many popular wireless communication systems, such as inter-vehicular communications, intelligent highway applications and mobile ad-hoc applications [3]. In contrast to conventional fixed-to-mobile (F2M) cellular radio systems, in M2M systems both the transmitter and receiver are in motion and equipped with low elevation antennas.The classical Rayleigh, Rician, or Nakagami fading channels have been found not to be applicable in M2M communication[4]. It has been observed that the effects of fading may be far severe than what can be modeled using the Nakagami distribution. Experimental results and theoretical analysis demonstrate that cascaded fading channels provide an accurate statistical model for M2M communication [5]. Cascaded Rayleigh (also named as $N$-Rayleigh) fading channel is presented in [6]. For $N=2$, this reduces to double-Rayleigh fading model in [7]. Moreover, this model has been extended to $N$-Nakagami fading model in [8]. For $N=2$, this reduces to double-Nakagami fading model in [9].

However, M2M communication may generate interference into the existing cellular network. The M2M transmit power and the distance between the users of a M2M pair needs to be constrained to have reliable cellular communication. A relay placed in between a M2M pair can extend the coverage area with less transmit power. This allows longer distance communication through M2M mode. Therefore, the relay-assisted M2M cooperative communication is attracting more and more attention. An approximation for average symbol error probability (ASEP) was derived for multiple-mobile-relay-based fixed-gain amplify-and-forward (FAF) relaying M2M cooperative networks over $N$-Nakagami fading channels in [10].

Some low-complexity cooperative diversity protocols such as selective relaying (SR) and incremental relaying (IR) schemes have been proposed for wireless cooperative networks. Taking the IR scheme into consideration, a new relaying scheme termed as incremental hybrid decode-amplify-forward (IHDAF) relaying was proposed in [11].

Multiple-input-multiple-output (MIMO) arises as a promising tool to enhance the reliability and capacity of wireless systems. However, multiple radio frequency chains must be implemented in MIMO systems, and it brings a corresponding increase in hardware complexity. In this situation, transmit antenna selection (TAS) arises as a practical way to reduce the system complexity. The exact closed-form expressions for outage probability (OP) of MIMO systems with two TAS selection schemes were derived in [12]. Both M2M communication and TAS are effective to improve the spectral efficiency and reduce the traffic load of the central network. However, to the best knowledge of the author, the OP performance of IHDAF relaying M2M networks with TAS and power allocation over $N$-Nakagami fading 
channels has not been investigated in the literature. Moreover, most results mentioned above do not take the power allocation into account. This is an important issue and will be discussed in this paper as it affects the OP performance. The main contributions are listed as follows:

1. Closed-form expressions are provided for the probability density function (PDF) and cumulative density functions (CDF) of the signal-to-noise ratio (SNR) over $N$-Nakagami fading channels. These are used to derive exact closed-form OP expressions for optimal and suboptimal TAS schemes. The derived OP expressions are in closed-form, which is very convenient to numerically and analytically handle.

2. A power allocation minimization problem is formulated to determine the optimum power distribution between the broadcasting and relaying phases.

3. The accuracy of the analytical results under different conditions is verified through numerical simulation. Results are presented which show that optimal TAS scheme has a better OP performance than suboptimal TAS scheme; the fading coefficient, the number of cascaded components, the relative geometrical gain, the number of transmit antennas, the number of receive antennas and the power-allocation parameter have an important influence on the OP performance.

4. The derived OP expressions can be used to evaluate the OP performance of the vehicular communication systems employed in inter-vehicular communications, intelligent highway applications and mobile ad-hoc applications.

The rest of the paper is organized as follows. Section 2 briefly reviews the research results related to this topic. The multiple-mobile-relay-based M2M system model is presented in Section 3. Section 4 provides the exact closed-form OP expressions for optimal TAS scheme. The exact closed-form OP expressions for suboptimal TAS scheme are derived in Section 5. In Section 6, the OP is optimized based on the power-allocation parameter. Section 7 conducts Monte Carlo simulations to verify the analytical results. Concluding remarks are given in Section 8.

\section{Related work}

Cooperative diversity has been recently proposed as an efficient solution to many challenging physical-layer problems in M2M communication field.Using FAF relaying, the pairwise error probability (PEP) of two relay-assisted vehicular scenarios over double-Nakagami fading channels was obtained in [13]. In [14], the OP performance of a mobile-relay-based two-way AF relaying system over $N$-Nakagami fading channels was investigated. The lower bound on OP of variable-gain AF relaying over $N$-Nakagami fading channels was investigated in [15]. By the moment generating function (MGF) method [16],the approximate ASEP performance of the mobile-relay-based M2M system over $N$-Nakagami fading channels was investigated in [17].

In regular cooperative-diversity networks, in addition to the direct link, all relays participate in sending the source signal to the destination. It wastes the channel resource because the relay forwards the signal every time regardless of the channel conditions. Since the relay and the source need to use orthogonal channels, additional resources will be used for relaying even if the relaying is not needed because the direct signal is good enough. To solve these problems, some low-complexity cooperative diversity protocols are proposed. Closed-form expressions for the error probability and average achievable rate of incremental DF (IDF) and incremental AF (IAF) relaying with a single relay over Rayleigh fading channels were derived in [18]. In [19], closed-form expressions for OP of IAF relaying M2M 
cooperative networks with relay selection over $N$-Nakagami fading channels were derived. Exact average bit error probability (BEP) expressions for IDF relaying M2M cooperative networks over $N$-Nakagami fading channels were derived in [20].

In [21], a novel cooperative diversity protocol called hybrid decode-amplify-forward (HDAF) was proposed. This protocol combined AF and SDF relaying. However, only the SEP performance was considered, and the analysis was based on the assumption that the relay can determine whether each received symbol was correctly detected or not, which was not practical in real systems. To provide a practical HDAF protocol, the forwarding decisions at the relay were based on the signal-to-noise ratio (SNR) of the received signal in [22], and closed-form expressions for the bit error probability of SNR-based HDAF relaying over independent non-identical flat Rayleigh fading channels with maximum ratio combining (MRC) were derived. Taking the IR scheme into consideration, a new relaying scheme termed as incremental HDAF (IHDAF) relaying was proposed in [11]. Closed-form expressions for the OP and bit error rate (BER) of the IHDAF relaying scheme were derived and presented over independent non-identical Rayleigh fading channels.

TAS can be introduced as a means to alleviate MIMO hardware complexity, while still retaining the diversity advantages. Owing to its simplicity and minimal communication requirements, many works has been done on TAS in MIMO systems. In [23], the performance of a dual-hop AF relay network with optimal and suboptimal TAS schemes was investigated. Closed-form expressions for OP and symbol error rate (SER) of TAS in multi-relay MIMO networks over Rician, Nakagami-m, Weibull, and generalized-K fading channels were derived in [24]. A good approximation of the distribution of the mutual information in the TAS system over Rayleigh fading channels was derived in [25], and it showed that channel hardening phenomenon was still retained as full complexity with antenna selection.

\section{The System and Channel Model}

\subsection{System Model}

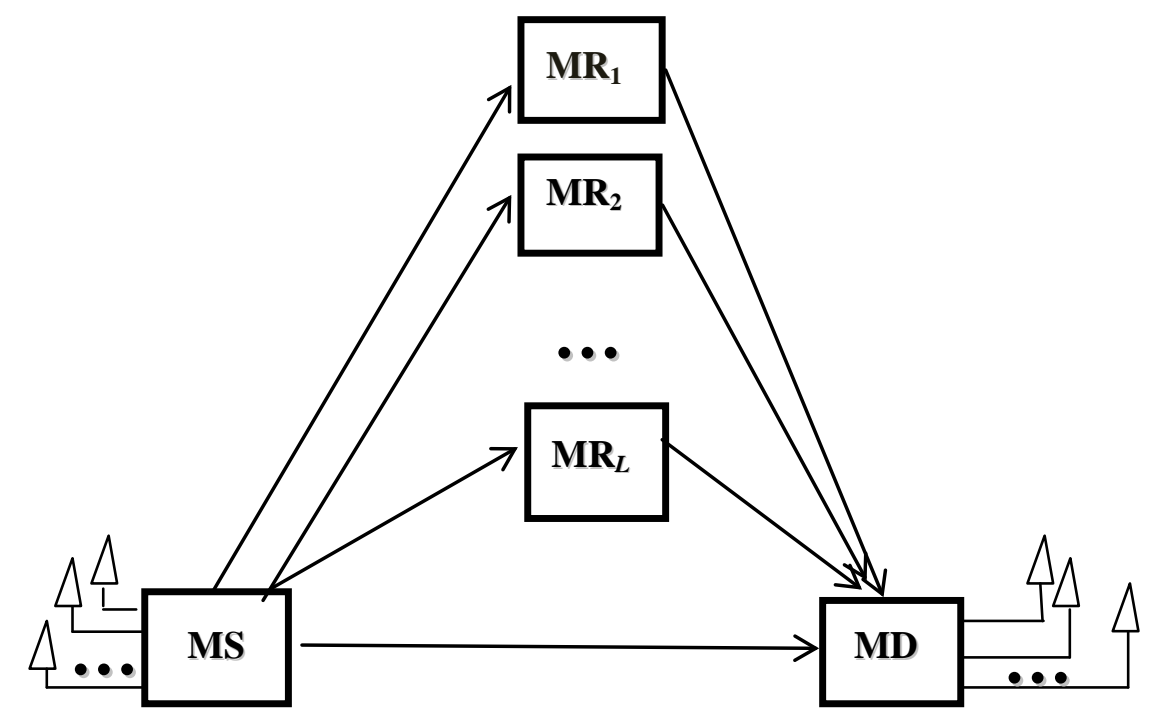

Fig. 1. The system model 
Consider a cooperation model shown in Fig. 1, namely a single mobile source (MS) node, $L$ mobile relay (MR) nodes, and a single mobile destination (MD) node. The nodes operate in half-duplex mode, MS is equipped with $N_{\mathrm{t}}$ antennas, MD is equipped with $N_{\mathrm{r}}$ antennas, whereas MR is equipped with a single antenna.

We assume that $N_{\mathrm{t}}$ antennas at MS and $N_{\mathrm{r}}$ antennas at MD have the same distance to the MR nodes. Using the approach in [13], the relative gain of the MS to MD link is $G_{\mathrm{SD}}=1$, the relative gain of the MS to $\mathrm{MR}_{l}$ link is $G_{\mathrm{SRl}}=\left(d_{\mathrm{SD}} / d_{\mathrm{SRl}}\right)^{v}$, and the relative gain of the $\mathrm{MR}_{l}$ to $\mathrm{MD}$ link is $G_{\mathrm{RD} l}=\left(d_{\mathrm{SD}} / d_{\mathrm{RD} l}\right)^{v}$, where $v$ is the path loss coefficient, and $d_{\mathrm{SD}}, d_{\mathrm{SRl}}$, and $d_{\mathrm{RD} l}$ represent the distances of the $\mathrm{MS}$ to $\mathrm{MD}, \mathrm{MS}$ to $\mathrm{MR}_{l}$, and $\mathrm{MR}_{l}$ to $\mathrm{MD}$ links, respectively [26]. To indicate the location of $\mathrm{MR}_{l}$ with respect to MS and MD, the relative geometrical gain $\mu_{l}=G_{\mathrm{SR} l} / G_{\mathrm{RD} l}$ (in decibels) is defined. When $\mathrm{MR}_{l}$ has the same distance to MS and $\mathrm{MD}, \mu_{l}$ is $1(0 \mathrm{~dB})$.When $\mathrm{MR}_{l}$ is close to $\mathrm{MD}, \mu_{l}$ has negative values. When $\mathrm{MR}_{l}$ is close to $\mathrm{MS}, \mu_{l}$ has positive values.

Let $\mathrm{MS}_{i}$ denote the $i$ th transmit antenna at $\mathrm{MS}, \mathrm{MD}_{j}$ denote the $j$ th receive antenna at $\mathrm{MD}$, so $h=h_{k}, k \in\left\{\right.$ SDij, SRil, RDlj\}, represent the complex channel coefficients of $\mathrm{MS}_{i} \rightarrow \mathrm{MD}_{j}$, $\mathrm{MS}_{i}$ $\rightarrow \mathrm{MR}_{l}$, and $\mathrm{MR}_{l} \rightarrow \mathrm{MD}_{j}$ links, respectively. Assume that the $i$ th antenna at MS is used to transmit the signal, during the first time slot, the received signal $r_{\mathrm{SD} i j}$ at $\mathrm{MD}_{j}$ can be written as

$$
r_{\mathrm{SD} i j}=\sqrt{K E} h_{\mathrm{SD} i j} x+n_{\mathrm{SD} i j}
$$

The received signal $r_{\mathrm{SR} i l}$ at $\mathrm{MR}_{l}$ can be written as

$$
r_{\mathrm{SR} i l}=\sqrt{G_{\mathrm{SRil}} K E} h_{\mathrm{SRil}} x+n_{\mathrm{SR} i l}
$$

where $x$ denotes the transmitted signal, $n_{\mathrm{SR} i l}$ and $n_{\mathrm{SD} i j}$ are the zero-mean complex Gaussian random variables with variance $N_{0} / 2$ per dimension. During two time slots, the total energy used by MS and MR is $E$. $K$ is the power-allocation parameter.

During the second time slot, only the best MR decides whether to forward the signal to the $\mathrm{MD}_{j}$ by comparing the instantaneous SNR $\gamma_{\mathrm{SD} i j}$ to a threshold $\gamma_{\mathrm{p}} . \gamma_{\mathrm{SD} i j}$ denotes the instantaneous SNR of the $\mathrm{MS}_{i}$ to $\mathrm{MD}_{j}$ link.

If $\gamma_{\mathrm{SD} i j}>\gamma_{\mathrm{p}}$, the $\mathrm{MD}_{j}$ will broadcast a 'success' message to the $\mathrm{MS}_{i}$ and the best MR. Then $\mathrm{MS}_{i}$ will transmit the next message, and the best MR remains silent.

If $\gamma_{\mathrm{SD} i j}<\gamma_{\mathrm{p}}$, the $\mathrm{MD}_{j}$ will broadcast a 'failure' message to the $\mathrm{MS}_{i}$ and the best MR. The best MR decides whether DF or AF cooperation is utilized to forward the received signal to the $\mathrm{MD}_{j}$ by comparing the instantaneous SNR $\gamma_{\mathrm{SR} i}$ to a threshold $\gamma_{\mathrm{T}}$, where $\gamma_{\mathrm{SR} i}$ represents the SNR of the link between $\mathrm{MS}_{i}$ and the best MR. The best MR is selected based on the following criterion

$$
\gamma_{\mathrm{SR} i}=\max _{1 \leq l \leq L}\left(\gamma_{\mathrm{SRil}}\right)
$$

where $\gamma_{\mathrm{SR} i l}$ represents the SNR of $\mathrm{MS}_{i} \rightarrow \mathrm{MR}_{l}$ link, and

$$
\gamma_{\text {SRil }}=\frac{K G_{\text {SRil }}\left|h_{\text {SRil }}\right|^{2} E}{N_{0}}=K G_{\text {SRil }}\left|h_{\text {SRil }}\right|^{2} \bar{\gamma}
$$

If $\gamma_{\mathrm{SRi}}>\gamma_{\mathrm{T}}$, the best MR then decodes and forwardes the signal $x_{\mathrm{r}}$ to $\mathrm{MD}_{j}$. Based on the DF cooperation protocol, the received signal at $\mathrm{MD}_{j}$ is given by

$$
r_{\mathrm{RDj}}=\sqrt{(1-K) G_{\mathrm{RDj}} E} h_{\mathrm{RDj}} X_{\mathrm{r}}+n_{\mathrm{RDj} j}
$$

where $n_{\mathrm{RDj}}$ is a conditionally zero-mean complex Gaussian random variable with variance $N_{0} / 2$ per dimension. 
If selection combining (SC) method is used at $\mathrm{MD}_{j}$, the output $\mathrm{SNR}$ can then be calculated as

$$
\gamma_{\mathrm{SC} i j}=\max \left(\gamma_{\mathrm{SDij}}, \gamma_{\mathrm{RDj}}\right)
$$

where $\gamma_{\mathrm{RDj}}$ represents the SNR of the link between the best MR and $\mathrm{MD}_{j}$.

If $\gamma_{\mathrm{SRi}}<\gamma_{\mathrm{T}}$, the best MR amplifies and forwards the signal to $\mathrm{MD}_{j}$. Based on the AF cooperation protocol, the received signal at $\mathrm{MD}_{j}$ is then given by

where

$$
r_{\mathrm{RD} i j}=\sqrt{c E} h_{\mathrm{SR} i} h_{\mathrm{RDj}} x+n_{\mathrm{RDj}}
$$

$$
c=\frac{K(1-K) G_{\mathrm{SR} i} G_{\mathrm{RD} j} \bar{\gamma}}{1+K G_{\mathrm{SR} i}\left|h_{\mathrm{SR} i}\right|^{2} \bar{\gamma}+(1-K) G_{\mathrm{RD} j}\left|h_{\mathrm{RD} j}\right|^{2} \bar{\gamma}}
$$

The output SNR of end-to-end link can then be calculated as

$$
\gamma_{\mathrm{SRDij}}=\frac{\gamma_{\mathrm{SRi}} \gamma_{\mathrm{RDj}}}{1+\gamma_{\mathrm{SRi}}+\gamma_{\mathrm{RDj}}}
$$

As far as we know, a convenient mathematical method to obtain PDF and CDF of $\gamma_{\text {SRDij }}$ exactly is still unachievable. Here, we adopt the method in [27] to obtain an approximate $\gamma_{\mathrm{SRD} i j}$. At high SNR, the $\gamma_{\text {SRDij }}$ can be approximated as

$$
\gamma_{\mathrm{SRDA} i j}=\frac{\gamma_{\mathrm{SRi}} \gamma_{\mathrm{RD} j}}{\gamma_{\mathrm{SR} i}+\gamma_{\mathrm{RD} j}}=\frac{1}{2} \frac{2}{\frac{1}{\gamma_{\mathrm{SR} i}}+\frac{1}{\gamma_{\mathrm{RD} j}}}
$$

By using the well-known inequality in [28], we can obtain an upper bound as

$$
\gamma_{\mathrm{SRDA} i j}<\gamma_{\mathrm{up} i j}=\min \left(\gamma_{\mathrm{SR} i}, \gamma_{\mathrm{RDj}}\right)
$$

If SC method is used at $\mathrm{MD}_{j}$, the output SNR can then be calculated as

$$
\gamma_{\mathrm{SCC} i j}=\max \left(\gamma_{\mathrm{SDij}}, \gamma_{\mathrm{upij}}\right)
$$

Using SC method at MD, the output SNR can then be calculated as

$$
\gamma_{\mathrm{SC}_{i}}=\max _{1 \leq j \leq N_{\mathrm{r}}}\left(\gamma_{i j}\right)
$$

where

$$
\gamma_{i j}=\left\{\begin{array}{cc}
\gamma_{\mathrm{SD} i j}, & \gamma_{\mathrm{SD} i j}>\gamma_{\mathrm{p}} \\
\gamma_{\mathrm{SC} i j}, & \gamma_{\mathrm{SD} i j}<\gamma_{\mathrm{p}}, \gamma_{\mathrm{SR} i}>\gamma_{\mathrm{T}} \\
\gamma_{\mathrm{SCC} i j}, & \gamma_{\mathrm{SD} i j}<\gamma_{\mathrm{p}}, \gamma_{\mathrm{SR} i}<\gamma_{\mathrm{T}}
\end{array}\right.
$$

The optimal TAS scheme should select the transmit antenna $w$ that maximizes the output SNR at MD, namely

$$
w=\max _{1 \leq i \leq N_{\mathrm{t}}}\left(\gamma_{\mathrm{SC}_{i}}\right)=\max _{1 \leq i \leq N_{\mathrm{t}}, 1 \leq j \leq N_{\mathrm{r}}}\left(\gamma_{i j}\right)
$$

The suboptimal TAS scheme is to select the transmit antenna that only maximizes the instantaneous SNR of the direct link $\mathrm{MS}_{i} \rightarrow \mathrm{MD}_{j}$, namely

$$
g=\max _{1 \leq i \leq N_{\mathrm{t}}, 1 \leq j \leq N_{\mathrm{r}}}\left(\gamma_{\mathrm{SD} i j}\right)
$$




\subsection{Channel model}

We assume that the links in the system are subject to independently and identically distributed (i.n.i.d) $N$-Nakagami fading. $h$ follows $N$-Nakagami distribution, which is given as[8]

$$
h=\prod_{t=1}^{N} a_{t}
$$

where $N$ is the number of cascaded components, $a_{t}$ is a Nakagami distributed random variable with PDF as

$$
f(a)=\frac{2 m^{m}}{\Omega^{m} \Gamma(m)} a^{2 m-1} \exp \left(-\frac{m}{\Omega} a^{2}\right)
$$

$\Gamma(\cdot)$ is the Gamma function, $m$ is the fading coefficient and $\Omega$ is a scaling factor.

With the aid of [8], the PDF of $h$ is given by

$$
f(h)=\frac{2}{h \prod_{t=1}^{N} \Gamma\left(m_{t}\right)} G_{0, N}^{N, 0}\left[\left.h^{2} \prod_{t=1}^{N} \frac{m_{t}}{\Omega_{t}}\right|_{m_{1}, \ldots, m_{N}}\right]
$$

where $\mathrm{G}[\cdot]$ is the Meijer's G-function[8].

Let $y=\left|h_{k}\right|^{2}$ represents the square of the amplitude of $h_{k}$. The corresponding CDF and PDF of $y$ can be given as [8]

$$
\begin{aligned}
& F(y)=\frac{1}{\prod_{t=1}^{N} \Gamma\left(m_{t}\right)} G_{1, N+1}^{N, 1}\left[\left.y \prod_{t=1}^{N} \frac{m_{t}}{\Omega_{t}}\right|_{m_{1}, \ldots, m_{N}, 0} ^{1}\right] \\
& f(y)=\frac{1}{y \prod_{t=1}^{N} \Gamma\left(m_{t}\right)} G_{0, N}^{N, 0}\left[\left.y \prod_{t=1}^{N} \frac{m_{t}}{\Omega_{t}}\right|_{m_{1}, \ldots, m_{N}}\right]
\end{aligned}
$$

\section{The OP of Optimal TAS Scheme}

The OP of optimal TAS scheme can be expressed as

$$
F_{\text {optimal }}=\operatorname{Pr}\left(\operatorname{maX}_{1 \leq i \leq N_{\mathrm{t}}, 1 \leq j \leq N_{\mathrm{r}}}\left(\gamma_{i j}\right)<\gamma_{\text {th }}\right)=\left(\operatorname{Pr}\left(\gamma_{i j}<\gamma_{\mathrm{th}}\right)\right)^{N_{\mathrm{t}} \times N_{\mathrm{r}}}
$$

$4.1 \gamma_{\mathrm{th}}<\gamma_{\mathrm{p}}$

When $\gamma_{\mathrm{p}}>\gamma_{\mathrm{th}}>\gamma_{\mathrm{T}}$, the OP of optimal TAS scheme can be expressed as

$$
\begin{aligned}
F_{\text {optimal }}= & \left(\operatorname{Pr}\left(\gamma_{\mathrm{SD}}<\gamma_{\mathrm{p}}, \gamma_{\mathrm{SR}}>\gamma_{\mathrm{T}}, \gamma_{\mathrm{SC}}<\gamma_{\mathrm{th}}\right)+\operatorname{Pr}\left(\gamma_{\mathrm{SD}}<\gamma_{\mathrm{p}}, \gamma_{\mathrm{SR}}<\gamma_{\mathrm{T}}, \gamma_{\mathrm{SCC}}<\gamma_{\mathrm{th}}\right)\right)^{N_{\mathrm{t}} \times N_{\mathrm{r}}} \\
= & \left(G_{1}+G_{2}\right)^{N_{\mathrm{t}} \times N_{\mathrm{r}}}
\end{aligned}
$$

where $\gamma_{\text {th }}$ is a given threshold for correct detection at MD.

The $G_{1}$ is evaluated as 


$$
\begin{aligned}
& G_{1}=\operatorname{Pr}\left(\gamma_{\mathrm{SD}}<\gamma_{\mathrm{p}}, \gamma_{\mathrm{SR}}>\gamma_{\mathrm{T}}, \gamma_{\mathrm{SC}}<\gamma_{\mathrm{th}}\right) \\
& =\operatorname{Pr}\left(\gamma_{\mathrm{SD}}<\gamma_{\mathrm{th}}, \gamma_{\mathrm{SR}}>\gamma_{\mathrm{T}}, \gamma_{\mathrm{RD}}<\gamma_{\mathrm{th}}\right) \\
& =\operatorname{Pr}\left(\gamma_{\mathrm{SD}}<\gamma_{\mathrm{th}}\right) \operatorname{Pr}\left(\gamma_{\mathrm{RD}}<\gamma_{\mathrm{th}}\right)\left(1-\operatorname{Pr}\left(\gamma_{\mathrm{SR}}<\gamma_{\mathrm{T}}\right)\right) \\
& \left.\left.=\frac{1}{\prod_{d=1}^{N} \Gamma\left(m_{d}\right)} G_{1, N+1}^{N, 1}\left[\left.\frac{\gamma_{\mathrm{th}}}{\overline{\gamma_{\mathrm{SD}}}} \prod_{d=1}^{N} \frac{m_{d}}{\Omega_{d}}\right|_{m_{1}, \ldots, m_{N}, 0} ^{1}\right] \times \frac{1}{\prod_{t t=1}^{N} \Gamma\left(m_{t t}\right)} G_{1, N+1}^{N, 1}\left[\left.\frac{\gamma_{\mathrm{th}}}{\overline{\gamma_{\mathrm{RD}}}} \prod_{t t=1}^{N} \frac{m_{t t}}{\Omega_{t t}}\right|_{m_{1}, \ldots, m_{N}, 0} ^{1}\right]\right)^{L}\right) \\
& \times\left(\frac{1-\left(\frac{1}{\prod_{t=1}^{N} \Gamma\left(m_{t}\right)} G_{1, N+1}^{N, 1}\left[\left.\frac{\gamma_{\mathrm{T}}}{\overline{\gamma_{\mathrm{SR}}}} \prod_{t=1}^{N} \frac{m_{t}}{\Omega_{t}}\right|_{m_{1}, \ldots, m_{N}, 0} ^{1}\right]\right)^{\frac{\gamma_{\mathrm{SD}}}{\gamma_{\mathrm{SR}}}}=K \bar{\gamma}}{\frac{\gamma_{\mathrm{r}}}{\gamma_{\mathrm{RD}}}}=(1-K) \bar{\gamma} G_{\mathrm{RD}} \bar{\gamma}\right.
\end{aligned}
$$

Next, the $G_{2}$ is evaluated.

$$
\begin{aligned}
& G_{2}=\operatorname{Pr}\left(\gamma_{\mathrm{SD}}<\gamma_{\mathrm{p}}, \gamma_{\mathrm{SR}}<\gamma_{\mathrm{T}}, \gamma_{\mathrm{SCC}}<\gamma_{\mathrm{th}}\right) \\
& =\operatorname{Pr}\left(\gamma_{\mathrm{SD}}<\gamma_{\mathrm{th}}, \gamma_{\mathrm{SR}}<\gamma_{\mathrm{T}}, \min \left(\gamma_{\mathrm{SR}}, \gamma_{\mathrm{RD}}\right)<\gamma_{\mathrm{th}}\right) \\
& =\operatorname{Pr}\left(\gamma_{\mathrm{SD}}<\gamma_{\mathrm{th}}\right) \operatorname{Pr}\left(\gamma_{\mathrm{SR}}<\gamma_{\mathrm{T}}\right)
\end{aligned}
$$$$
=\frac{1}{\prod_{d=1}^{N} \Gamma\left(m_{d}\right)} G_{1, N+1}^{N, 1}\left[\left.\frac{\gamma_{\mathrm{th}}}{\overline{\gamma_{\mathrm{SD}}}} \prod_{d=1}^{N} \frac{m_{d}}{\Omega_{d}}\right|_{m_{1}, \ldots, m_{N}, 0} ^{1}\right] \times\left(\frac{1}{\prod_{t=1}^{N} \Gamma\left(m_{t}\right)} G_{1, N+1}^{N, 1}\left[\left.\frac{\gamma_{\mathrm{T}}}{\overline{\gamma_{\mathrm{SR}}}} \prod_{t=1}^{N} \frac{m_{t}}{\Omega_{t}}\right|_{m_{1}, \ldots, m_{N}, 0} ^{1}\right]\right)^{L}
$$

$4.2 \gamma_{\mathrm{th}}>\gamma_{\mathrm{p}}$

When $\gamma_{\text {th }}>\gamma_{\mathrm{p}}, \gamma_{\text {th }}>\gamma_{\mathrm{T}}$, the OP of optimal TAS scheme can be expressed as

$$
\begin{aligned}
F_{\text {optimal }}= & \left(\begin{array}{l}
\operatorname{Pr}\left(\gamma_{\mathrm{p}}<\gamma_{\mathrm{SD}}<\gamma_{\mathrm{th}}\right)+\operatorname{Pr}\left(\gamma_{\mathrm{SD}}<\gamma_{\mathrm{p}}, \gamma_{\mathrm{SR}}>\gamma_{\mathrm{T}}, \gamma_{\mathrm{SC}}<\gamma_{\mathrm{th}}\right)+ \\
\operatorname{Pr}\left(\gamma_{\mathrm{SD}}<\gamma_{\mathrm{p}}, \gamma_{\mathrm{SR}}<\gamma_{\mathrm{T}}, \gamma_{\mathrm{SCC}}<\gamma_{\mathrm{th}}\right)
\end{array}\right)^{N_{\mathrm{t}} \times N_{\mathrm{r}}} \\
= & \left(I_{1}+I_{2}+I_{3}\right)^{N_{\mathrm{t}} \times N_{\mathrm{r}}}
\end{aligned}
$$

The $I_{1}$ is given as

$$
\begin{aligned}
& I_{1}=\operatorname{Pr}\left(\gamma_{\mathrm{p}}<\gamma_{\mathrm{SD}}<\gamma_{\mathrm{th}}\right) \\
& =\frac{1}{\prod_{d=1}^{N} \Gamma\left(m_{d}\right)}\left(G_{1, N+1}^{N, 1}\left[\left.\frac{\gamma_{\mathrm{th}}}{\overline{\gamma_{\mathrm{SD}}}} \prod_{d=1}^{N} \frac{m_{d}}{\Omega_{d}}\right|_{m_{1}, \ldots, m_{N}, 0} ^{1}\right]-G_{1, N+1}^{N, 1}\left[\left.\frac{\gamma_{\mathrm{p}}}{\overline{\gamma_{\mathrm{SD}}}} \prod_{d=1}^{N} \frac{m_{d}}{\Omega_{d}}\right|_{m_{1}, \ldots, m_{N}, 0} ^{1}\right]\right)
\end{aligned}
$$


The $I_{2}$ is evaluated as

$$
\begin{aligned}
& I_{2}=\operatorname{Pr}\left(\gamma_{\mathrm{SD}}<\gamma_{\mathrm{p}}\right) \operatorname{Pr}\left(\gamma_{\mathrm{RD}}<\gamma_{\mathrm{th}}\right)\left(1-\operatorname{Pr}\left(\gamma_{\mathrm{SR}}<\gamma_{\mathrm{T}}\right)\right) \\
= & \frac{1}{\prod_{d=1}^{N} \Gamma\left(m_{d}\right)} G_{1, N+1}^{N, 1}\left[\left.\frac{\gamma_{\mathrm{p}}}{\overline{\gamma_{\mathrm{SD}}}} \prod_{d=1}^{N} \frac{m_{d}}{\Omega_{d}}\right|_{m_{1}, \ldots, m_{N}, 0} ^{1}\right] \times \frac{1}{\prod_{t t=1}^{N} \Gamma\left(m_{\mathrm{tt}}\right)} G_{1, N+1}^{N, 1}\left[\left.\frac{\gamma_{\mathrm{th}}}{\gamma_{\mathrm{RD}}} \prod_{t t=1}^{N} \frac{m_{\mathrm{tt}}}{\Omega_{t t}}\right|_{m_{1}, \ldots, m_{N}, 0} ^{1}\right] \\
& \times\left(1-\left(\frac{1}{\prod_{t=1}^{N} \Gamma\left(m_{t}\right)} G_{1, N+1}^{N, 1}\left[\left.\frac{\gamma_{\mathrm{T}}}{\gamma_{\mathrm{SR}}} \prod_{t=1}^{N} \frac{m_{t}}{\Omega_{t}}\right|_{m_{1}, \ldots, m_{N}, 0} ^{1}\right]\right)^{L}\right) \\
I_{3}= & \operatorname{Pr}\left(\gamma_{\mathrm{SD}}<\gamma_{\mathrm{p}}, \gamma_{\mathrm{SR}}<\gamma_{\mathrm{T}}, \gamma_{\mathrm{SCC}}<\gamma_{\mathrm{th}}\right) \\
= & \operatorname{Pr}\left(\gamma_{\mathrm{SD}}<\gamma_{\mathrm{p}}, \gamma_{\mathrm{SR}}<\gamma_{\mathrm{T}}, \min \left(\gamma_{\mathrm{SR}}, \gamma_{\mathrm{RD}}\right)<\gamma_{\mathrm{th}}\right) \\
= & \operatorname{Pr}\left(\gamma_{\mathrm{SD}}<\gamma_{\mathrm{p}}\right) \operatorname{Pr}\left(\gamma_{\mathrm{SR}}<\gamma_{\mathrm{T}}\right) \\
= & \frac{1}{\prod_{d=1}^{N} \Gamma\left(m_{d}\right)} G_{1, N+1}^{N, 1}\left[\left.\frac{\gamma_{\mathrm{p}}}{\overline{\gamma_{\mathrm{SD}}}} \prod_{d=1}^{N} \frac{m_{d}}{\Omega_{d}}\right|_{m_{1}, \ldots, m_{N}, 0} ^{1}\right] \times\left(\frac{1}{\prod_{t=1}^{N} \Gamma\left(m_{t}\right)} G_{1, N+1}^{N, 1}\left[\left.\frac{\gamma_{\mathrm{T}}}{\underline{\gamma_{\mathrm{SR}}}} \prod_{t=1}^{N} \frac{m_{t}}{\Omega_{t}}\right|_{m_{1}, \ldots, m_{N}, 0} ^{1}\right]\right)^{L}
\end{aligned}
$$

\section{The OP of Suboptimal TAS Scheme}

$5.1 \gamma_{\mathrm{th}}<\gamma_{\mathrm{p}}$

When $\gamma_{\mathrm{p}}>\gamma_{\mathrm{th}}>\gamma_{\mathrm{T}}$, the OP of suboptimal TAS scheme can be expressed as

$$
\begin{gathered}
F_{\text {suboptimal }}=\operatorname{Pr}\left(\gamma_{\mathrm{SDg}}<\gamma_{\mathrm{th}}, \gamma_{\mathrm{SR}}<\gamma_{\mathrm{T}}\right)+\operatorname{Pr}\left(\gamma_{\mathrm{SDg}}<\gamma_{\mathrm{th}}, \gamma_{\mathrm{SR}}>\gamma_{\mathrm{T}}, \gamma_{\mathrm{RD}}<\gamma_{\mathrm{th}}\right) \\
=G G_{1}+G G_{2}
\end{gathered}
$$

where

$$
\gamma_{\mathrm{SDg}}=\max _{1 \leq i \leq N_{\mathrm{t}}, 1 \leq j \leq N_{\mathrm{r}}}\left(\gamma_{\mathrm{SDij}}\right)
$$

The $G G_{1}$ is evaluated as

$$
\begin{aligned}
& G G_{1}=\left(\operatorname{Pr}\left(\gamma_{\mathrm{SD}}<\gamma_{\mathrm{th}}\right)\right)^{N_{\mathrm{t}} \times N_{\mathrm{r}}} \times \operatorname{Pr}\left(\gamma_{\mathrm{SR}}<\gamma_{\mathrm{T}}\right) \\
& =\left(\frac{1}{\prod_{d=1}^{N} \Gamma\left(m_{d}\right)} G_{1, N+1}^{N, 1}\left[\left.\frac{\gamma_{\mathrm{th}}}{\underline{\gamma_{\mathrm{SD}}}} \prod_{d=1}^{N} \frac{m_{d}}{\Omega_{d}}\right|_{m_{1}, \ldots, m_{N}, 0} ^{1}\right]\right)^{N_{\mathrm{t}} \times N_{\mathrm{r}}} \times\left(\frac{1}{\prod_{t=1}^{N} \Gamma\left(m_{t}\right)} G_{1, N+1}^{N, 1}\left[\left.\frac{\gamma_{\mathrm{T}}}{\gamma_{\mathrm{SR}}} \prod_{t=1}^{N} \frac{m_{t}}{\Omega_{t}}\right|_{m_{1}, \ldots, m_{N}, 0} ^{1}\right]\right)^{L}
\end{aligned}
$$

Next, the $G G_{2}$ is evaluated. 


$$
\begin{aligned}
& G G_{2}=\left(\operatorname{Pr}\left(\gamma_{\mathrm{SD}}<\gamma_{\mathrm{th}}\right)\right)^{N_{\mathrm{t}} \times N_{\mathrm{r}}} \times \operatorname{Pr}\left(\gamma_{\mathrm{RD}}<\gamma_{\mathrm{th}}\right) \times\left(1-\operatorname{Pr}\left(\gamma_{\mathrm{SR}}<\gamma_{\mathrm{T}}\right)\right) \\
& =\left(\frac{1}{\prod_{d=1}^{N} \Gamma\left(m_{d}\right)} G_{1, N+1}^{N, 1}\left[\left.\frac{\gamma_{\mathrm{th}}}{\gamma_{\mathrm{SD}}} \prod_{d=1}^{N} \frac{m_{d}}{\Omega_{d}}\right|_{m_{1}, \ldots, m_{N}, 0} ^{1}\right]\right)^{N_{\mathrm{t}} \times N_{\mathrm{r}}} \times \\
& \frac{1}{\prod_{t t=1}^{N} \Gamma\left(m_{t t}\right)} G_{1, N+1}^{N, 1}\left[\left.\frac{\gamma_{\mathrm{th}}}{\overline{\gamma_{\mathrm{RD}}}} \prod_{t t=1}^{N} \frac{m_{t t}}{\Omega_{t t}}\right|_{m_{1}, \ldots, m_{N}, 0} ^{1}\right] \times \\
& \left(1-\left(\frac{1}{\prod_{t=1}^{N} \Gamma\left(m_{t}\right)} G_{1, N+1}^{N, 1}\left[\left.\frac{\gamma_{\mathrm{T}}}{\overline{\gamma_{\mathrm{SR}}}} \prod_{t=1}^{N} \frac{m_{t}}{\Omega_{t}}\right|_{m_{1}, \ldots, m_{N}, 0} ^{1}\right]\right)^{L}\right)
\end{aligned}
$$

$5.2 \gamma_{\mathrm{th}}>\gamma_{\mathrm{p}}$

When $\gamma_{\text {th }}>\gamma_{\mathrm{p}}, \gamma_{\text {th }}>\gamma_{\mathrm{T}}$, the OP of suboptimal TAS scheme can be expressed as

$$
\begin{aligned}
F_{\text {suboptimal }} & =\operatorname{Pr}\left(\gamma_{\mathrm{p}}<\gamma_{\mathrm{SDg}}<\gamma_{\mathrm{th}}\right)+\operatorname{Pr}\left(\gamma_{\mathrm{SDg}}<\gamma_{\mathrm{p}}, \gamma_{\mathrm{SR}}<\gamma_{\mathrm{T}}\right)+\operatorname{Pr}\left(\gamma_{\mathrm{SDg}}<\gamma_{\mathrm{p}}, \gamma_{\mathrm{SR}}>\gamma_{\mathrm{T}}, \gamma_{\mathrm{RD}}<\gamma_{\mathrm{th}}\right) \\
& =I I_{1}+I I_{2}+I I_{3}
\end{aligned}
$$

The $I I_{1}$ is given as

$$
\begin{aligned}
& I_{1}=\left(\operatorname{Pr}\left(\gamma_{\mathrm{p}}<\gamma_{\mathrm{SDg}}<\gamma_{\mathrm{th}}\right)\right) \\
& =\left(\frac{1}{\prod_{d=1}^{N} \Gamma\left(m_{d}\right)} G_{1, N+1}^{N, 1}\left[\frac{\gamma_{\mathrm{th}}}{\overline{\gamma_{\mathrm{SD}}}} \prod_{d=1}^{N} \frac{m_{d}}{\Omega_{d}}||_{m_{1}, \ldots, m_{N}, 0}^{1}\right]\right)^{N_{\mathrm{t}} \times N_{\mathrm{r}}} \\
& -\left(\frac{1}{\prod_{d=1}^{N} \Gamma\left(m_{d}\right)} G_{1, N+1}^{N, 1}\left[\left.\frac{\gamma_{\mathrm{p}}}{\overline{\gamma_{\mathrm{SD}}}} \prod_{d=1}^{N} \frac{m_{d}}{\Omega_{d}}\right|_{m_{1}, \ldots, m_{N}, 0} ^{1}\right]\right)^{N_{\mathrm{t}} \times N_{\mathrm{r}}}
\end{aligned}
$$

Next, the $I_{2}$ is evaluated.

$$
\begin{aligned}
& I I_{2}=\operatorname{Pr}\left(\gamma_{\mathrm{SDg}}<\gamma_{\mathrm{p}}, \gamma_{\mathrm{SR}}<\gamma_{\mathrm{T}}\right) \\
& =\left(\frac{1}{\prod_{d=1}^{N} \Gamma\left(m_{d}\right)} G_{1, N+1}^{N, 1}\left[\left.\frac{\gamma_{\mathrm{p}}}{\overline{\gamma_{\mathrm{SD}}}} \prod_{d=1}^{N} \frac{m_{d}}{\Omega_{d}}\right|_{m_{1}, \ldots, m_{N}, 0} ^{1}\right]\right)^{N_{\mathrm{t}} \times N_{\mathrm{r}}} \times\left(\frac{1}{\prod_{t=1}^{N} \Gamma\left(m_{t}\right)} G_{1, N+1}^{N, 1}\left[\left.\frac{\gamma_{\mathrm{T}}}{\overline{\gamma_{\mathrm{SR}}}} \prod_{t=1}^{N} \frac{m_{t}}{\Omega_{t}}\right|_{m_{1}, \ldots, m_{N}, 0} ^{1}\right]\right)^{L}
\end{aligned}
$$




$$
\begin{aligned}
& I I_{3}=\operatorname{Pr}\left(\gamma_{\mathrm{SDg}}<\gamma_{\mathrm{p}}, \gamma_{\mathrm{SR}}>\gamma_{\mathrm{T}}, \gamma_{\mathrm{RD}}<\gamma_{\mathrm{th}}\right) \\
& =\left(\frac{1}{\prod_{d=1}^{N} \Gamma\left(m_{d}\right)} G_{1, N+1}^{N, 1}\left[\left.\frac{\gamma_{\mathrm{p}}}{\overline{\gamma_{\mathrm{SD}}}} \prod_{d=1}^{N} \frac{m_{d}}{\Omega_{d}}\right|_{m_{1}, \ldots ., m_{N}, 0} ^{1}\right]\right)^{N_{\mathrm{t}} \times N_{\mathrm{r}}} \times \\
& \frac{1}{\prod_{t t=1}^{N} \Gamma\left(m_{t t}\right)} G_{1, N+1}^{N, 1}\left[\left.\frac{\gamma_{\mathrm{th}}}{\overline{\gamma_{\mathrm{RD}}}} \prod_{t t=1}^{N} \frac{m_{t t}}{\Omega_{t t}}\right|_{m_{1}, \ldots, m_{N}, 0} ^{1}\right] \times \\
& \left(1-\left(\frac{1}{\prod_{t=1}^{N} \Gamma\left(m_{t}\right)} G_{1, N+1}^{N, 1}\left[\left.\frac{\gamma_{\mathrm{T}}}{\overline{\gamma_{\mathrm{SR}}}} \prod_{t=1}^{N} \frac{m_{t}}{\Omega_{t}}\right|_{m_{1}, \ldots, m_{N}, 0} ^{1}\right]\right)^{L}\right)
\end{aligned}
$$

\section{ABEP-Optimized Power Allocation}

To further improve the performance, we aim to optimally allocate the power between broadcasting and relaying phases. For optimization of the power allocation, we consider OP as our objective function. The resulting $\mathrm{OP}$ needs to be minimized with respect to the power-allocation parameter $K$.

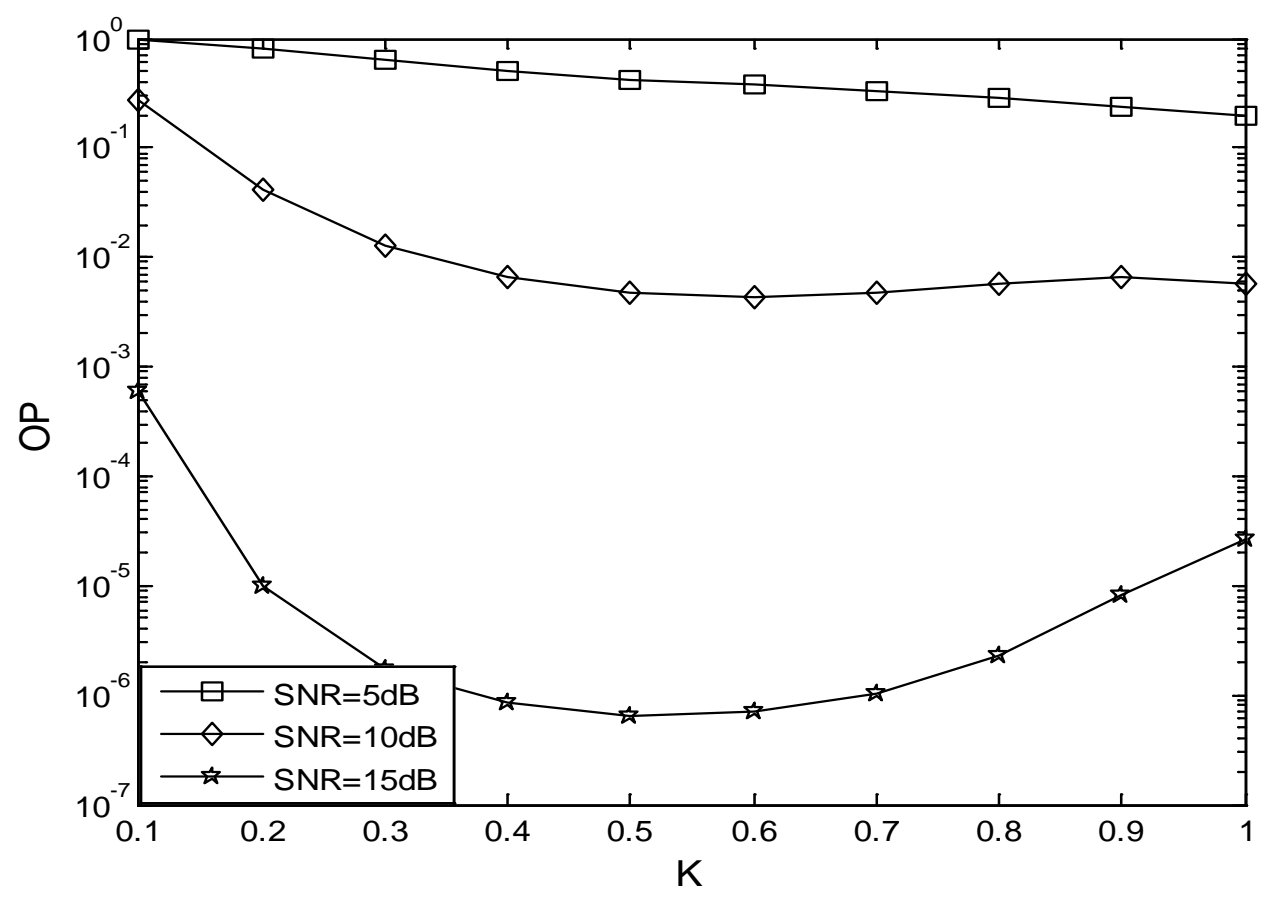

Fig. 2. The effect of the power-allocation parameter $K$ on the OP performance 
Fig. 2 presents the effect of the power-allocation parameter $K$ on the OP performance when $\gamma_{\mathrm{th}}=5 \mathrm{~dB}, \gamma_{\mathrm{T}}=2 \mathrm{~dB}, \gamma_{\mathrm{p}}=6 \mathrm{~dB}$. The number of cascaded components is $N=2$. The fading coefficient is $m=2$. The relative geometrical gain is $\mu=0 \mathrm{~dB}$. The number of transmit antennas is $N_{\mathrm{t}}=2$. The number of mobile relays is $L=2$. The number of receive antennas is $N_{\mathrm{r}}=2$. Simulation results show that the OP performance is improved with the SNR increased. For example, when $K=0.7$, the $\mathrm{OP}$ is $3.3 \times 10^{-1}$ with $\mathrm{SNR}=5 \mathrm{~dB}, 4.6 \times 10^{-3}$ with $\mathrm{SNR}=10 \mathrm{~dB}, 1.0 \times 10^{-6}$ with $S N R=15 \mathrm{~dB}$. When $S N R=5 \mathrm{~dB}$, the optimum value of $K$ is $0.99 ; \mathrm{SNR}=10 \mathrm{~dB}$, the optimum value of $K$ is 0.60 ; $\mathrm{SNR}=15 \mathrm{~dB}$, the optimum value of $K$ is 0.52 . This indicates that the equal power allocation (EPA) scheme is not the best scheme.

Unfortunately, an analytical solution for power allocation values in the general case is very difficult. We resort to numerical methods to solve this optimization problem. The optimum power allocation (OPA) values can be obtained a priori for given values of operating SNR and propagation parameters. The OPA values can be stored for use as a lookup table in practical implementation.

In Table 1, we present optimum values of $K$ with the relative geometrical gain $\mu$. The number of cascaded components is $N=2$, the fading coefficient is $m=2$, the relative geometrical gain is $\mu=5 \mathrm{~dB}, 0 \mathrm{~dB},-5 \mathrm{~dB}$, the number of transmit antennas is $N_{\mathrm{t}}=2$, the number of mobile relays is $L=2$, the number of receive antennas is $N_{\mathrm{r}}=2$, and the given threshold is $\gamma_{\mathrm{th}}=5 \mathrm{~dB}$, $\gamma_{\mathrm{T}}=2 \mathrm{~dB}, \gamma_{\mathrm{p}}=6 \mathrm{~dB}$. For example, when $\mu=5 \mathrm{~dB}$, the SNR is low, nearly all the power should be used in broadcast phase. As the SNR increased, the optimum values of $K$ are reduced, and more than $50 \%$ of the power should be used in broadcast phase.

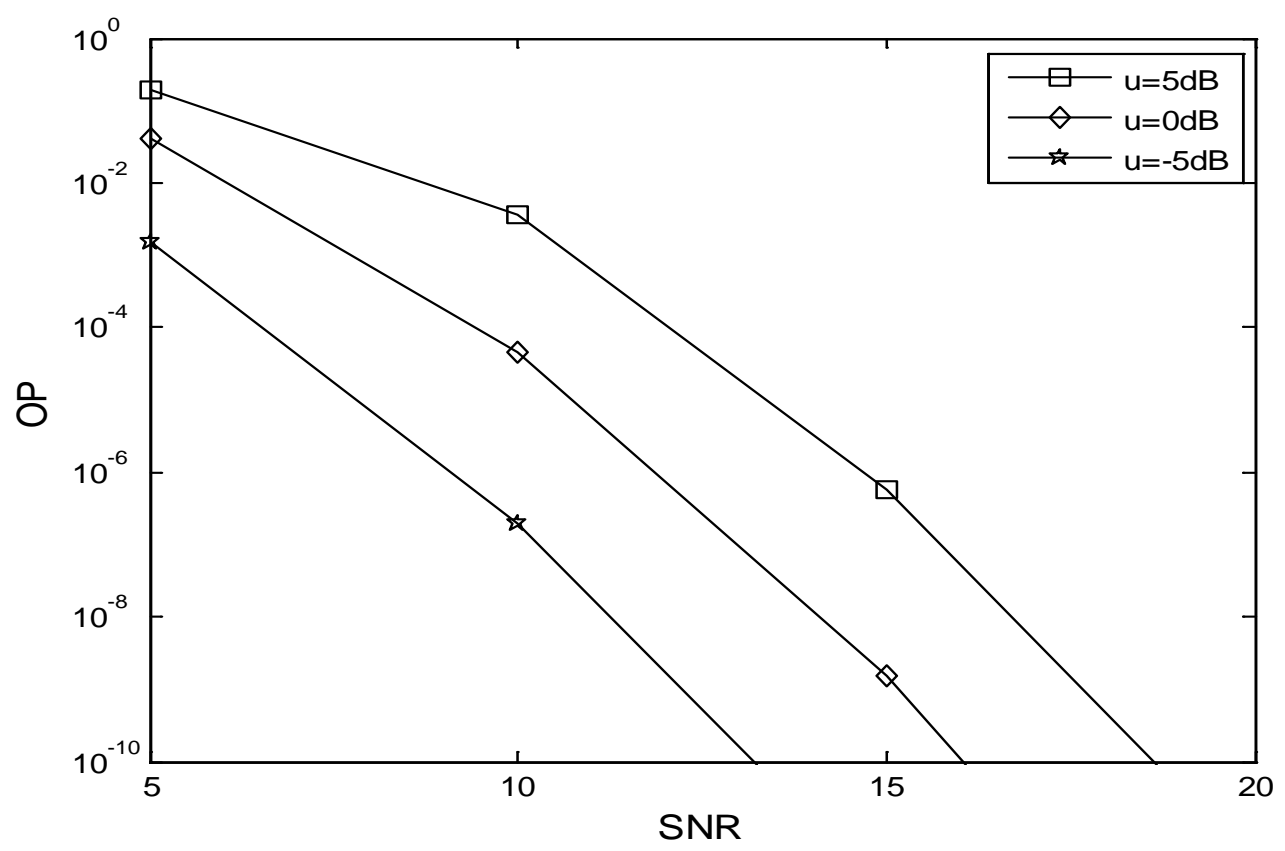

Fig. 3. The effect of the relative geometrical gain $\mu$ on the OP performance

Fig. 3 presents the effect of the relative geometrical gain $\mu$ on the OP performance when $\gamma_{\mathrm{th}}=5 \mathrm{~dB}, \gamma_{\mathrm{T}}=2 \mathrm{~dB}, \gamma_{\mathrm{p}}=6 \mathrm{~dB}$. The values of $K$ are used in Table 1 . Simulation results show that the OP performance is improved as $\mu$ reduced. For example, when SNR $=10 \mathrm{~dB}$, the OP is $3.6 \times 10^{-3}$ for $\mu=5 \mathrm{~dB}, 4.5 \times 10^{-5}$ for $\mu=0 \mathrm{~dB}$, and $1.9 \times 10^{-7}$ for $\mu=-5 \mathrm{~dB}$. This indicates that the best location for the relay is near the destination. For a fixed $\mu$, an increase in the SNR reduces the 
OP.

Table 1. OPA parameters $K$

\begin{tabular}{|c|c|c|c|}
\hline SNR & $\mu=5 \mathrm{~dB}$ & $\mu=0 \mathrm{~dB}$ & $\mu=-5 \mathrm{~dB}$ \\
\hline 5 & 0.99 & 0.41 & 0.51 \\
\hline 10 & 0.51 & 0.41 & 0.47 \\
\hline 15 & 0.50 & 0.44 & 0.45 \\
\hline 20 & 0.50 & 0.47 & 0.46 \\
\hline
\end{tabular}

\section{Numerical Results}

In this section, we present Monte-Carlo simulations to confirm the derived analytical results. The simulation results are obtained for BPSK modulations. Additionally,random number simulation was done to confirm the validity of the analytical approach. All the computations were done in Matlab and some of the integrals were verified through Maple. The links between $\mathrm{MS} \rightarrow \mathrm{MD}, \mathrm{MS} \rightarrow \mathrm{MR}$ and $\mathrm{MR} \rightarrow \mathrm{MD}$ are modeled as $N$-Nakagami distribution. The total energy is $E=1$. The fading coefficient is $m=1,2,3$, the number of cascaded components is $N=2$, 3 , 4, the number of mobile relays is $L=2$, the number of receive antennas is $N_{\mathrm{r}}=2$, and the number of transmit antennas is $N_{\mathrm{t}}=1,2,3$, respectively.

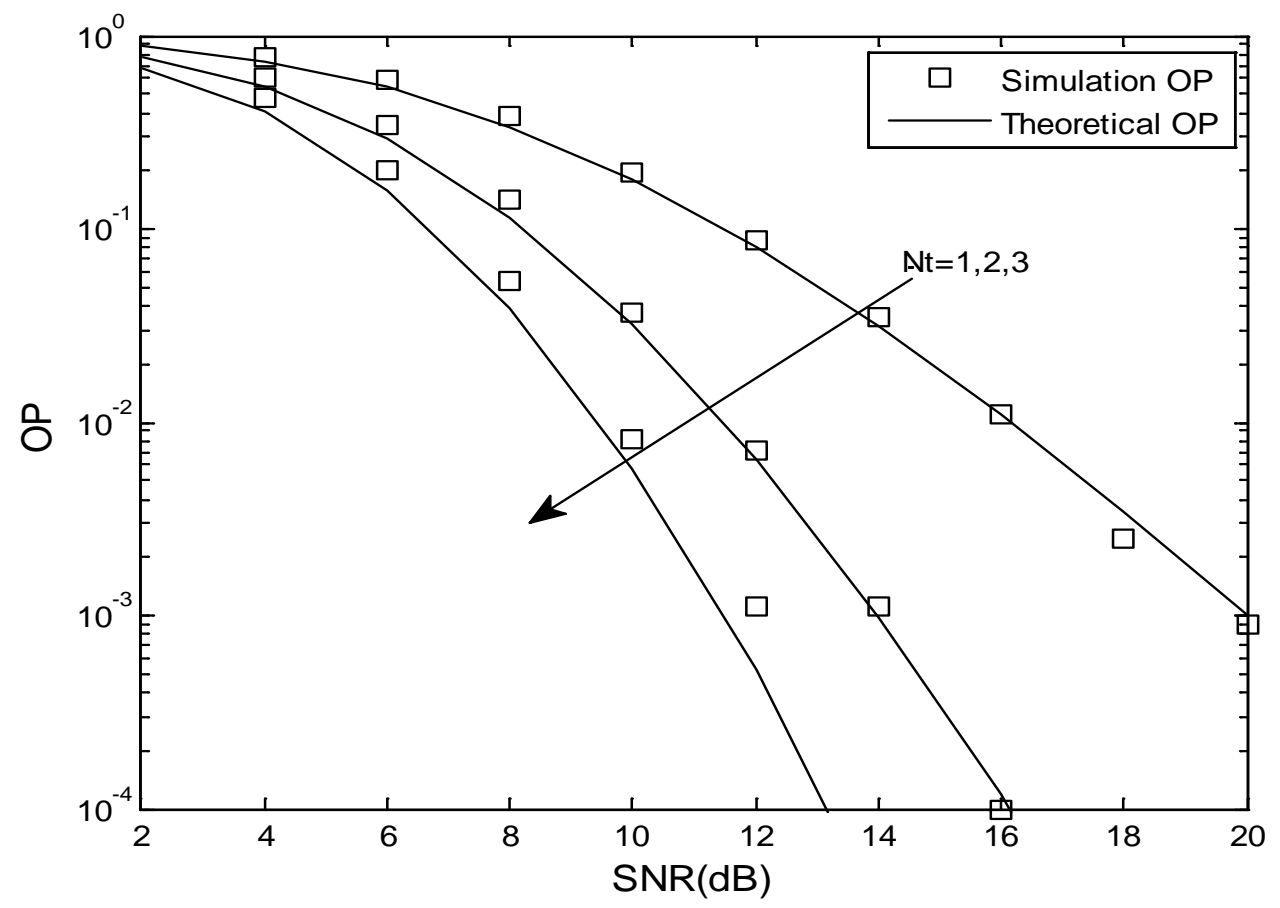

Fig. 4. The OP performance of optimal TAS scheme when $\gamma_{\mathrm{th}}<\gamma_{\mathrm{p}}$

Fig. 4 presents the OP performance of optimal TAS scheme when $\gamma_{\mathrm{th}}=5 \mathrm{~dB}, \gamma_{\mathrm{T}}=2 \mathrm{~dB}$, $\gamma_{\mathrm{P}}=6 \mathrm{~dB}$. Fig. 5 presents the OP performance of optimal TAS scheme when $\gamma_{\mathrm{th}}=5 \mathrm{~dB}, \gamma_{\mathrm{T}}=2 \mathrm{~dB}$, $\gamma_{\mathrm{p}}=2 \mathrm{~dB}$. The number of cascaded components is $N=2$. The fading coefficient is $m=1$. The power-allocation parameter is $K=0.5$. The number of transmit antennas is $N_{\mathrm{t}}=1,2,3$. The number of mobile relays is $L=2$. The number of receive antennas is $N_{\mathrm{r}}=2$. The relative geometrical gain is $\mu=0 \mathrm{~dB}$. In order to verify our analytical results, we have also plotted 
simulation based results. We see that the analytical results match perfectly with the simulations. As expected, the $\mathrm{OP}$ is improved as the number of transmit antennas increased. For example, when $\gamma_{\mathrm{th}}>\gamma_{\mathrm{p}}, \mathrm{SNR}=10 \mathrm{~dB}, N_{\mathrm{t}}=1$, the OP is $2.2 \times 10^{-1}, N_{\mathrm{t}}=2$, the OP is $5.0 \times 10^{-2}, N_{\mathrm{t}}=3$, the OP is $1.1 \times 10^{-2}$. With $N_{\mathrm{t}}$ fixed, an increase in the SNR decreases the OP.

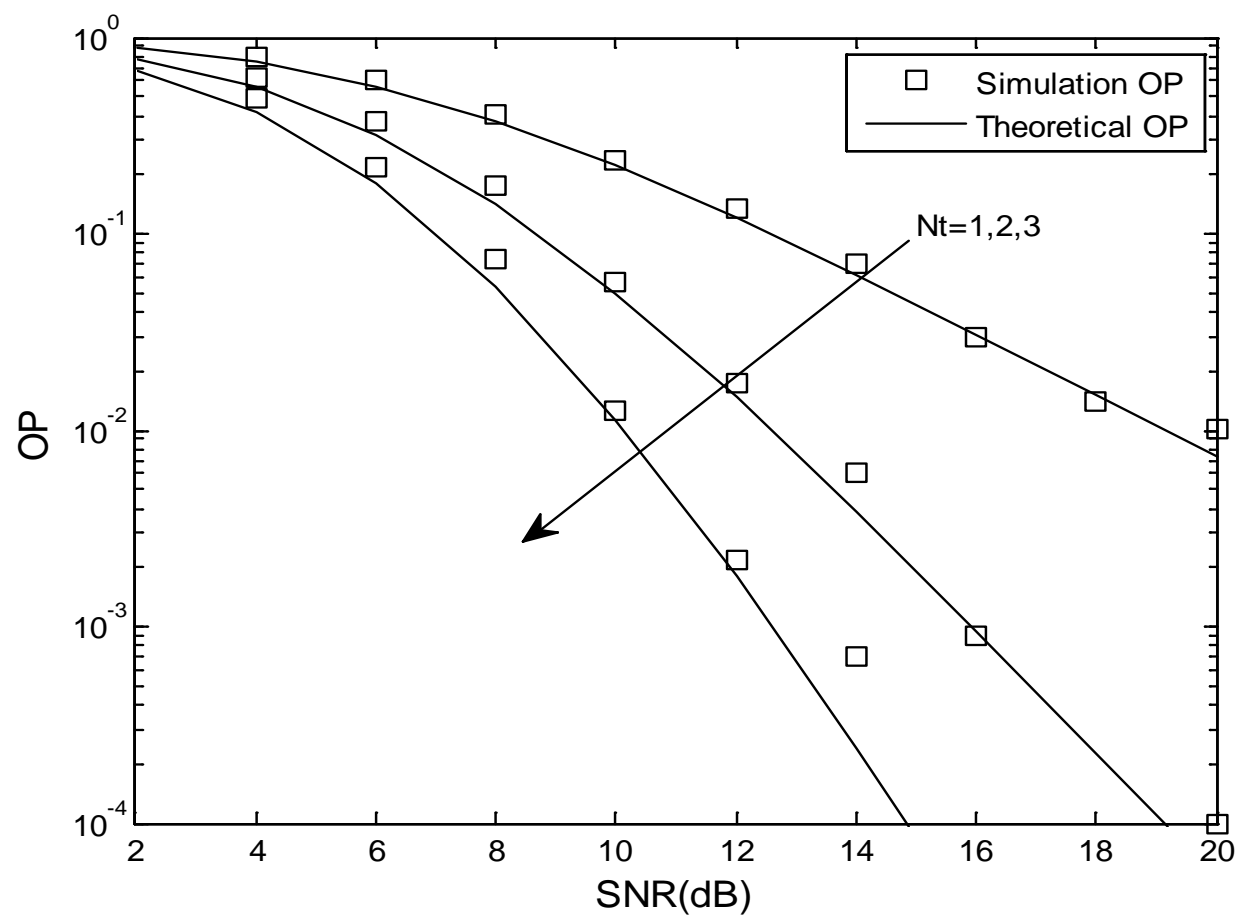

Fig. 5. The OP performance of optimal TAS scheme when $\gamma_{\mathrm{th}}>\gamma_{\mathrm{p}}$

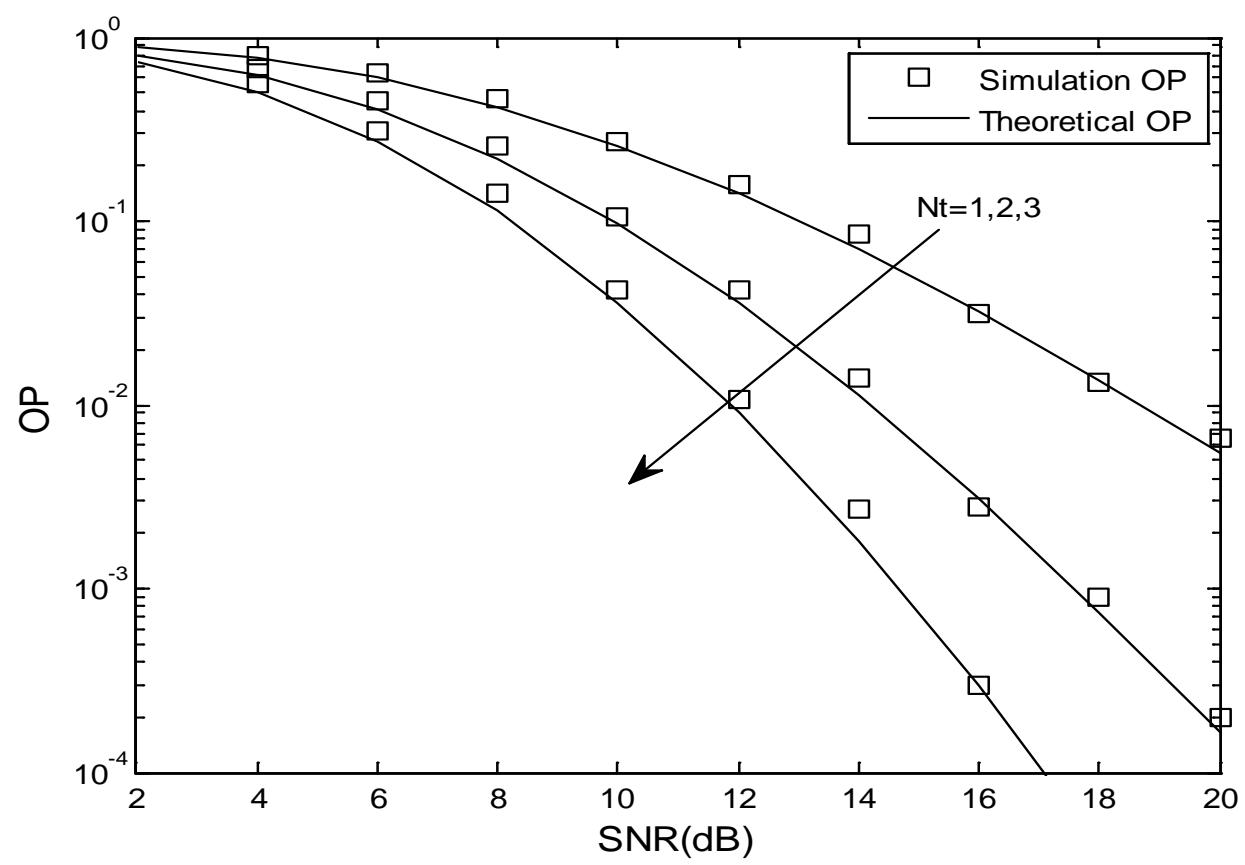

Fig. 6. The OP performance of suboptimal TAS scheme when $\gamma_{\mathrm{th}}<\gamma_{\mathrm{p}}$ 


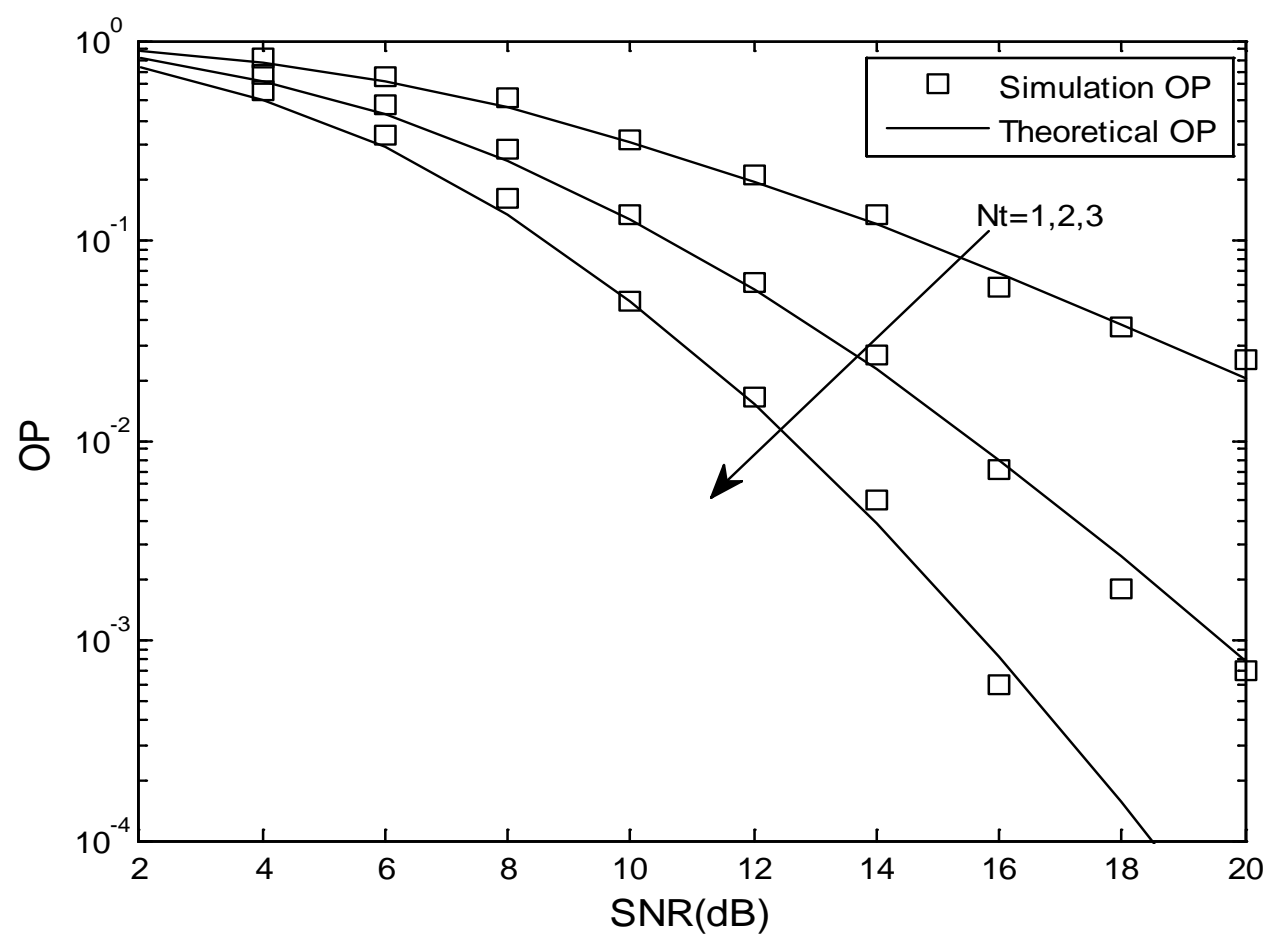

Fig. 7. The OP performance of suboptimal TAS scheme when $\gamma_{\mathrm{th}}<\gamma_{\mathrm{p}}$

Fig. 6 presents the OP performance of suboptimal TAS scheme when $\gamma_{\mathrm{th}}=5 \mathrm{~dB}, \gamma_{\mathrm{r}}=2 \mathrm{~dB}$, $\gamma_{\mathrm{p}}=6 \mathrm{~dB}$. Fig. 7 presents the OP performance of suboptimal TAS scheme when $\gamma_{\mathrm{th}}=5 \mathrm{~dB}$, $\gamma_{\mathrm{T}}=2 \mathrm{~dB}, \gamma_{\mathrm{p}}=2 \mathrm{~dB}$. The number of cascaded components is $N=2$. The fading coefficient is $m=1$. The power-allocation parameter is $K=0.5$. The number of transmit antennas is $N_{\mathrm{t}}=1,2,3$. The number of mobile relays is $L=2$. The number of receive antennas is $N_{\mathrm{r}}=2$. The relative geometrical gain is $\mu=0 \mathrm{~dB}$. In order to verify our analytical results, we have also plotted simulation based results. From Fig. 6 and Fig. 7, we can obtain that the analytical results match perfectly with the simulations. As expected, the OP is improved as the number of transmit antennas increased. For example, when $\gamma_{\mathrm{th}}=5 \mathrm{~dB}, \gamma_{\mathrm{T}}=2 \mathrm{~dB}, \gamma_{\mathrm{p}}=2 \mathrm{~dB}, \mathrm{SNR}=12 \mathrm{~dB}, N_{\mathrm{t}}=1$, the OP is $2.0 \times 10^{-1}, N_{\mathrm{t}}=2$, the OP is $5.7 \times 10^{-2}, N_{\mathrm{t}}=3$, the OP is $1.5 \times 10^{-2}$. With $N_{\mathrm{t}}$ fixed, an increase in the SNR decreases the OP.

In Fig. 8, we compare the theoretical OP performance of optimal TAS scheme and suboptimal TAS scheme by varying the number of antennas $N_{\mathrm{t}}$, when $\gamma_{\mathrm{th}}=5 \mathrm{~dB}, \gamma_{\mathrm{T}}=2 \mathrm{~dB}$, $\gamma_{\mathrm{p}}=2 \mathrm{~dB}$. The number of cascaded components is $N=2$. The fading coefficient is $m=1$. The power-allocation parameter is $K=0.5$.The relative geometrical gain is $\mu=0 \mathrm{~dB}$. The number of transmit antennas is $N_{\mathrm{t}}=2,3$. The number of mobile relays is $L=2$. The number of receive antennas is $N_{\mathrm{r}}=2$. To avoid clutter, we have not plotted the simulation based results. In all cases, as expected, when $N_{\mathrm{t}}$ is fixed, optimal TAS scheme has a better OP performance in all SNR regimes. As predicted by our analysis, the performance gap between two TAS schemes decreases when $N_{\mathrm{t}}$ is increased. When the SNR is low, the OP performance gap between optimal TAS scheme with $N_{\mathrm{t}}=2$ and suboptimal TAS scheme with $N_{\mathrm{t}}=3$ is negligible, as the SNR increases, the OP performance gap also increases. 


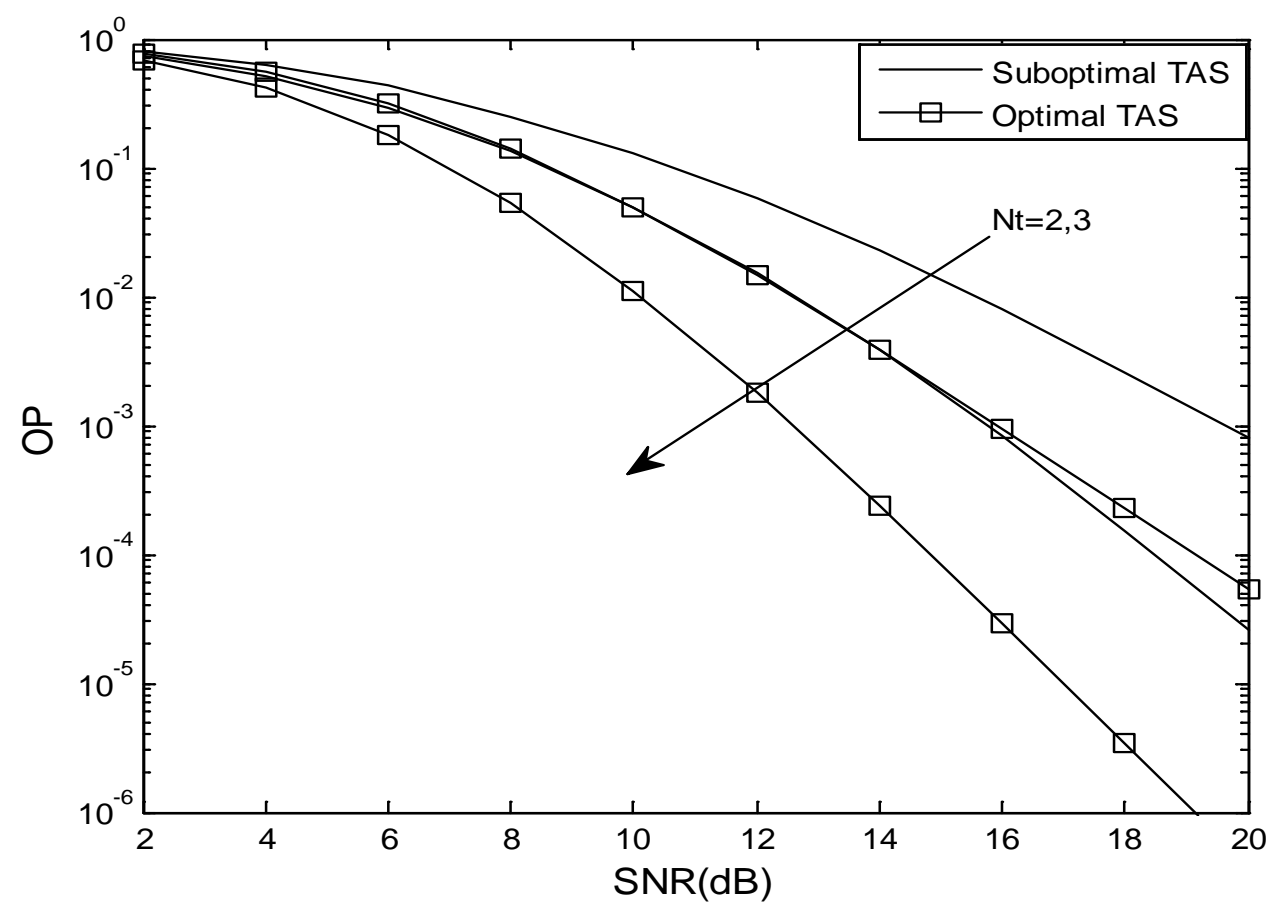

Fig. 8. The OP performance comparison of two TAS schemes

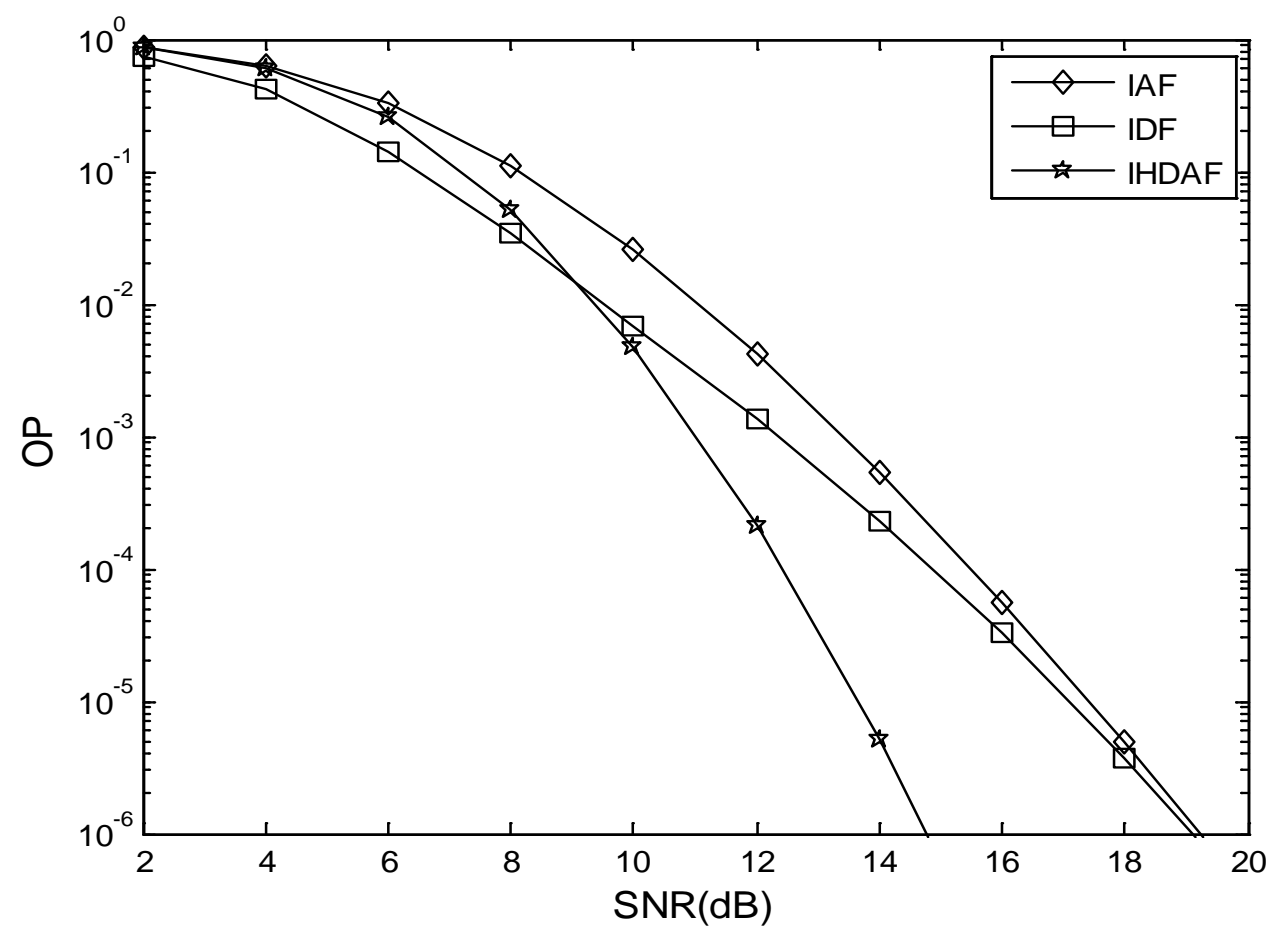

Fig. 9. The OP performance comparison of three relaying schemes 
Fig. 9 illustrates the OP performance of IAF, IDF, IHDAF three relaying schemes. The number of cascaded components is $N=2$. The fading coefficient is $m=2$. The power-allocation parameter is $K=0.5$. The relative geometrical gain is $\mu=0 \mathrm{~dB}$. The number of transmit antennas is $N_{\mathrm{t}}=2$. The number of mobile relays is $L=2$. The number of receive antennas is $N_{\mathrm{r}}=2$. The given threshold is $\gamma_{\mathrm{th}}=5 \mathrm{~dB}, \gamma_{\mathrm{T}}=2 \mathrm{~dB}, \gamma_{\mathrm{p}}=6 \mathrm{~dB}$. In all cases, as expected, IDF scheme has a better OP performance than IAF scheme in all SNR regimes. When the SNR is low, IDF scheme outperforms the other two schemes significantly. With the SNR increases, IHDAF scheme outperforms the other two schemes significantly.

\section{Conclusion}

The exact closed-form OP expressions for IHDAF relaying M2M networks with TAS over $\mathrm{N}$-Nakagami fading channels are derived in this paper. The simulation results show that optimal TAS scheme has a better OP performance than suboptimal TAS scheme; the power-allocation parameter $K$ has an important influence on the OP performance. Expressions were derived which can be used to evaluate the OP performance of vehicular communication systems employed in inter-vehicular, intelligent highway and mobile ad-hoc applications.

\section{References}

[1] G. Wu, S. Talwar, K. Johnsson, N. Himayat, and K. D. Johnson, "M2M: From mobile to embedded internet," IEEE Communications Magazine, vol. 49, no. 4, pp. 36-43, April, 2011. Article (CrossRef Link)

[2] M. Jo, T. Maksymyuk, B. Strykhalyuk, C.H. Cho, "Device-to-device based heterogeneous radio access network architecture for mobile cloud computing,” IEEE Wireless Communications, vol.22, no.3 , pp. 50-58, June, 2015. Article (CrossRef Link)

[3] S. Mumtaz, K. M. S. Huq, and J. Rodriguez, "Direct mobile-to-mobile communication: paradigm for 5G,” IEEE Wireless Communications, vol.21, no.5, pp. 14-23, May, 2014. Article (CrossRef Link)

[4] X. Cheng, C.X. Wang, D.I. Laurenson, S.Salous, A.V.Vasilakos, “An adaptive geometry-based stochastic model for non-isotropic MIMO mobile-to-mobile channels," IEEE Transactions on Wireless Communications, vol.8, no.9, pp.4824-4835, September, 2009. Article (CrossRef Link)

[5] J. Salo, H. E. Sallabi, and P. Vainikainen, "Statistical analysis of the multiple scattering radio channel,” IEEE Transactions on Antennas Propagation, vol. 54, no. 11 ,pp. 3114-3124, November , 2006. Article (CrossRef Link)

[6] J. Salo, H. E. Sallabi, and P. Vainikainen, "The distribution of the product of independent Rayleigh random variables,” IEEE Transactions on Antennas and Propagation, vol. 54, no.2, pp. 639-643, February, 2006. Article (CrossRef Link)

[7] M. Uysal, "Diversity analysis of space-time coding in cascaded Rayleigh fading channels,” IEEE Communications Letters, vol. 10, no. 3, pp. 165-167, March, 2006.Article (CrossRef Link)

[8] G. K. Karagiannidis, N. C. Sagias, and P. T. Mathiopoulos, "N*Nakagami: A novel stochastic model for cascaded fading channels,” IEEE Transactions on Communications, vol. 55, no. 8, pp. 1453-1458, August, 2007. Article (CrossRef Link)

[9] F. K. Gong, P. Ye, Y. Wang, and N. Zhang, "Cooperative mobile-to-mobile communications over double Nakagami-m fading channels,” IET Communications, vol. 6, no. 18, pp. 3165-3175, December, 2012. Article (CrossRef Link) 
[10] L.W. Xu, H. Zhang, X. Liu and T. A. Gulliver, "Performance analysis of FAF relaying M2M cooperative networks over $N$-Nakagami fading channels,” International Journal of Signal Processing, Image Processing and Pattern Recognition, vol.8, no. 5, pp. 249-258, May, 2015. Article (CrossRef Link)

[11] Z.Q. Bai, J.L. Jia, C.X. Wang, and D.F. Yuan, "Performance analysis of SNR-Based incremental hybrid decode-amplify-forward cooperative Relaying Protocol," IEEE Transactions on Communications, vol. 63, no. 6, pp. 2094-2106, June , 2015. Article (CrossRef Link)

[12] L. Yang, "MIMO systems with transmit antenna selection and power allocation over correlated channels," Wireless Personal Communications, vol. 55, no. 2, pp. 225-235, February, 2010. Article (CrossRef Link)

[13] H. Ilhan, M. Uysal, and I. Altunbas, "Cooperative diversity for intervehicular communication: Performance analysis and optimization,” IEEE Transactions on Vehicular Technology, vol. 58, no. 7, pp. 3301-3310, July, 2009. Article (CrossRef Link)

[14] C.Zhang, J.Ge, J.Li, Y.Hu, "Performance analysis for mobile-relay-based M2M two-way AF relaying in N*Nakagami-m fading,” Electronics Letters, vol.49, no.5, pp.344-346, February, 2013. Article (CrossRef Link)

[15] L.W. Xu, H. Zhang, T.T. Lu and T. A. Gulliver, "Outage probability analysis of the VAF relaying M2M networks,” International Journal of Hybrid Information Technology, vol. 8, no.5, pp. 357-366, May, 2015. Article (CrossRef Link)

[16] F.Yilmaz, M.S.Alouini, “A unified MGF-Based capacity analysis of diversity combiners over generalized fading channels,” IEEE Transactions on Communications, vol.60, no.3, pp.862-875, March, 2012. Article (CrossRef Link)

[17] L.W. Xu, H. Zhang, T.T. Lu and X. Liu, “The approximate ASEP of the mobile-relay-based M2M system,” International Journal of Electrical Engineering, vol. 22, no. 1, pp. 1-7, January ,2015. Article (CrossRef Link)

[18] S.S.Ikki, M.H.Ahmed, "Performance analysis of incremental-relaying cooperative-diversity networks over Rayleigh fading channels,” IET Communications, vol. 5, no. 3, pp. 337-349, March, 2011. Article (CrossRef Link)

[19] L.W. Xu, H. Zhang, T.T. Lu, and T. A. Gulliver, "Performance analysis of the IAF relaying M2M cooperative networks over N-Nakagami fading channels,” Journal of Communications, vol. 10, no. 3, pp.185-191, March, 2015. Article (CrossRef Link)

[20] L.W. Xu, H. Zhang, and T. A. Gulliver, "Performance analysis of IDF relaying M2M cooperative networks over $N$-Nakagami fading channels,” KSII Transactions on Internet \& Information Systems, vol. 9, no. 10, pp. 3983-4001, Octorber, 2015. Article (CrossRef Link)

[21] T. Q. Duong, and H. J. Zepernick, “On the performance gain of hybrid decode-amplify-forward cooperative communications,” EURASIP Journal on Wireless Communications and Networking, vol. 2009, Article ID 479463, 10 pages, 2009. Article (CrossRef Link)

[22] H. Chen, J. Liu, C. Zhai, and L. Zheng, "Performance analysis of SNR-based hybrid decode-amplify-forward cooperative diversity networks over Rayleigh fading channels,” in Proc. of IEEE Conf. on Wireless Communications and Networking, pp. 1-6, April 18-21, 2010. Article (CrossRef Link)

[23] H. A. Suraweera, P. J. Smithnd, A. Nallanathan, and J. S. Thompson, “Amplify-and-forward relaying with optimal and suboptimal transmit antenna selection,” IEEE Transactions on Wireless Communications, vol. 10, no. 6, pp. 1874-1885, June, 2011. Article (CrossRef Link)

[24] P. L. Yeoh, M. Elkashlan, N. Yang, D. B. D. Costa, and T. Q. Duong, "Energy efficiency of large-scale multiple antenna systems with transmit antenna selection," IEEE Transactions on Vehicular Technology, vol. 62, no. 2, pp. 933-939, February, 2013. Article (CrossRef Link) 
[25] H. Li, L.Y. Song, M. Debbah, "Unified analysis of transmit antenna selection in MIMO multi-relay networks," IEEE Transactions on Communications, vol. 62, no. 2, pp. 638-647, February, 2014. Article (CrossRef Link)

[26] H. Ochiai, P. Mitran, and V. Tarokh, "Variable-rate two-phase collaborative communication protocols for wireless networks," IEEE Transactions on Vehicular Technology, vol. 52, no. 9, pp. 4299-4313, September, 2006. Article (CrossRef Link)

[27] M. O. Hasna, and M. S. Alouini, "Harmonic mean and end-to-end performance of transmission systems with relays,” IEEE Transactions on Wireless Communications, vol.52, no. 1, pp. 130-135, January, 2004. Article (CrossRef Link)

[28] P. A. Anghel, and M. Kaveh, "Exact symbol error probability of a cooperative network in Rayleigh-fading environment,” IEEE Transactions on Wireless Communications, vol. 3, no. 5, pp. 1416-1421, May, 2004. Article (CrossRef Link)

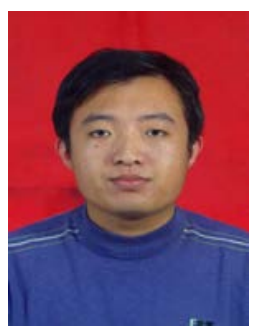

Lingwei Xu received his B.E. degree in Communication Engineering from Qingdao Technological University, P.R. China in 2011. He received his M.E. degree in Electronics and Communication Engineering from Ocean University of China, P.R. China in 2013. Now he is a Ph.D. candidate in Ocean University of China. His research interests include ultra-wideband radio systems, MIMO wireless systems, and M2M wireless communications.

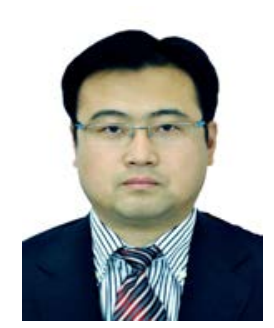

Hao Zhang received his B.E. degree in Telecommunications Engineering and Industrial Management from Shanghai Jiaotong University, P.R.China in 1994. He received his MBA degree from New York Institute of Technology, USA in 2001. He received his Ph.D. degree in Electrical and Computer Engineering from the University of Victoria, Canada in 2004. He is currently a professor in the Department of Electronic Engineering at Ocean University of China and an adjunct professor in the Department of Electrical and Computer Engineering at the University of Victoria. His research interests include ultra-wideband radio systems, MIMO wireless systems, cooperative communication networks

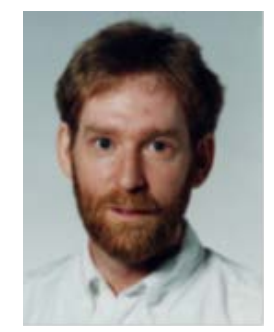

T. Aaron Gulliver received his Ph.D. degree in Electrical and Computer Engineering from the University of Victoria, Victoria, BC, Canada in 1989. He is a professor in the Department of Electrical and Computer Engineering. From 1989 to 1991 he was employed as a Defense Scientist at Defense Research Establishment Ottawa, Ottawa, ON, Canada. He has held academic positions at Carleton University, Ottawa, and the University of Canterbury, Christchurch, New Zealand. He joined the University of Victoria in 1999 and is a Professor in the Department of Electrical and Computer Engineering. In 2002 he became a Fellow of the Engineering Institute of Canada, and in 2012 a Fellow of the Canadian Academy of Engineering. He is also a senior member of IEEE. His research interests include information theory and communication theory, and ultra wideband communication. 